\title{
Editorials
}

\section{Physical health in severe mental illness}

The indivisible self has become divided. Divided by the structures of medical specialism, which themselves reflect the understanding of a wider society. Divided too by a mind and body philosophy that prefers reductionism to holism. More recently, divided by healthcare management struggling to address the issues of integrated care for patients who place multiple physical and mental healthcare demands upon a system so often seeming to be at breaking point.

In spite of forces that divide the self, recent years have seen some convergence in physical and mental health care. In psychiatry, there has been an increasing awareness of the need to address the physical health issues of patients. Primary care too has adopted a more holistic agenda, promoting the concept of 'medical generalism', encompassing the medical, psychological, and social agenda of patients.

\section{THE SIZE OF THE MORTALITY GAP}

The mortality gap for patients with schizophrenia has been apparent since the 17 th century in observations on the mortality of 'lunacy'. 'A gap of about 20 years for males and 15 years for females is still experienced by patients with psychosis in the developed world. ${ }^{2}$ Reporting a somewhat smaller mortality gap of 13 years for males and 12 years for females, Chang et al found that schizophrenia, schizoaffective disorder, and bipolar disorder contributed similarly to observed excess mortality values, whereas patients with substance use disorders had a larger mortality gap.

\section{THE CAUSE OF THE MORTALITY GAP}

The causes of the mortality gap can broadly be divided into four categories. First, patients with serious mental illness (SMI) have a higher rate of suicide, accidental, or violent death. Second, several risk factors combine to adversely affect health such as smoking, lower levels of exercise, and obesity (which itself may be the iatrogenic consequences of antipsychotic medication). These, in turn, increase the rates of cardiovascular disease, chronic obstructive pulmonary disease (COPD), and cancers (in addition to the cancer risk associated with smoking, we now know that obesity contributes to 11 types of cancer): ${ }^{4}$ they are also linked to social deprivation and the broader determinants of SMI. Third,

In spite of forces that divide the self, recent years have seen some convergence in physical and mental health care.

antipsychotic medication itself adds to the burden of long-term conditions (LTCs) mediated by iatrogenic weight gain, hyperlipidaemia, and diabetes. Finally, patients with SMI experience reduced access to health care either through delayed presentation, reduced uptake of health screening and preventive care, difficulty coping with the demands of LTC monitoring and treatment, or misattribution of symptoms. Both patients and health professionals may interpret symptoms of physical disease, possibly even red-flag symptoms, as just another manifestation of their SMI, a process known as diagnostic overshadowing'. Finally, stigmatising attitudes towards people with severe mental illnesses, held by healthcare professionals, may play a significant role.

Of all the causes of the mortality gap, mental health services often focus on the less common causes of suicide and violent death. In fact, excess mortality is predominantly the result of cardiovascular respiratory, or malignant disease.

\section{COMPARISONS WITH OTHER MORTALITY GAPS}

The importance of the SMI mortality gap can be seen by comparing it with that for other conditions commonly within the remit of GPs. For example, the mortality gap attributable to smoking has been put at 2 to 3 years in the US or around 7 years in New Zealand. ${ }^{3}$ For type 2 diabetes the gap has been estimated at 1.3 to 2.0 years (for males and females, respectively).

\section{DIRECTION OF THE MORTALITY GAP}

It might be reasonable to assume that with improvements in psychiatric care, in medical care, and in the wealth of society, the SMI mortality gap would slowly decrease. Evidence is now emerging that, far from narrowing, the gap has widened. In a 30-year study from Denmark, mortality in the general population had fallen substantially such that mean life expectancy increased over the study period by 6.3 years. ${ }^{6}$ In contrast, over the same period life expectancy in the population with schizophrenia declined by 1.3 years. These findings were accompanied by much concern that observed deterioration had occurred in a society with a strong healthcare system and pride in the equality of its social structures. One possible explanation offered was that closure of inpatient psychiatric facilities had not been accompanied by parallel increases in the provision of community mental health services. In a UK study over the period 2000 to 2014, all-cause mortality for patients with SMI fell. For patients with bipolar disorder, the decline was at a similar rate to the overall population rate of decline. For patients with schizophrenia, the decline was slower, thus resulting in a widening of the mortality gap.?

\section{CHALLENGES OF ADDRESSING THE PHYSICAL HEALTH OF PATIENTS WITH SMI}

Shared physical health care of patients with SMI between primary and community services is likely to become increasingly common, driven both by patient choice lsome patients may choose only to access care through their GP, and others only through mental health services) and relatively recent National Institute for Health and Care Excellence (NICE) guidance, which recommends that the mental health team should maintain responsibility for 


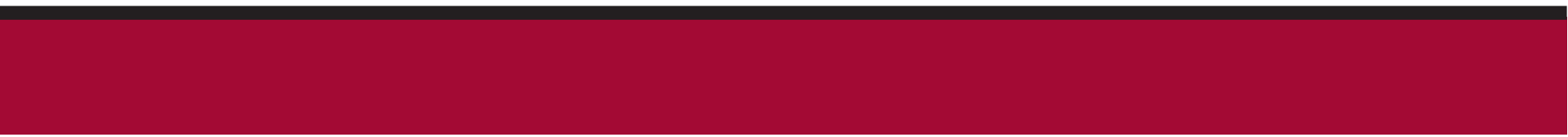

"... treating tobacco dependence is effective in patients with SMI ... smoking rates are three times that of the general population ...

\section{ADDRESS FOR CORRESPONDENCE}

\section{Mark Ashworth}

School of Population Sciences and Health Services Research, Faculty of Life Sciences and Medicine, King's College London, 5th Floor Addison House, Guy's Campus, London, SE1 1UL, UK.

\section{E-mail: mark.ashworthakcl.ac.uk}

monitoring users' physical health for at least 12 months after starting antipsychotic medication. ${ }^{8}$ In practice, health promotion and LTC management are core competencies for GPs who are likely to play a leading role in addressing physical health issues in patients with SMI. The Quality and Outcomes Framework (QOF) is unlikely to offer a sufficient infrastructure for guiding physical health care, especially since serum $\mathrm{HbA1c}$ and cholesterol monitoring were removed as SMI indicators in April 2014. The King's Fund has highlighted the importance of prioritising the needs of patients with $\mathrm{SMI}$ at clinical commissioning group (CCG) level with the provision of tailored services for long-term conditions and for smoking cessation. ${ }^{9}$ In other words, instead of a focus on achieving overall population targets for cardiovascular health, pulmonary rehabilitation, and smoking cessation, for example, the focus needs to shift to patients with SMI to ensure that they are not excluded from quality targets.

We know that smoking is the largest cause of preventable death in the UK. The situation is no different for patients with SMI. However, bespoke smoking cessation services for patients with SMI are not widely available and interventions have been hampered by lingering concern that treating nicotine addiction might have adverse effects on the underlying mental state. A survey of the literature concluded that treating tobacco dependence is effective in patients with SMI and that treatments are equally effective as for the general population; moreover, there was no evidence of worsening mental state in those with 'stable psychiatric conditions'. ${ }^{10}$
Efforts to address smoking cessation are all the more urgent in patients with SMI because smoking rates are three times that of the general population and, at the same time, these patients are less likely to receive smoking cessation support. ${ }^{9}$ Here is a clear opportunity for primary care to make a difference.

The ultimate challenge is to achieve parity. Parity of physical health care in patients with and without SMI. Primary care in the UK is well positioned to narrow that yawning (and possibly widening) gap through its focus on whole-person care. The divided self may be a little less divided if patients with SMI can access more holistic care.

\section{Mark Ashworth}

Reader in Primary Care, School of Population Sciences and Health Services Research, Faculty of Life Sciences and Medicine, King's College London, London.

\section{Peter Schofield,}

Wolfson Lecturer in Population Sciences, School of Population Sciences and Health Services Research, Faculty of Life Sciences and Medicine, King's College London, London.

\section{Jayati Das-Munshi,}

Clinician Scientist/Honorary Consultant Psychiatrist, Academy of Medical Sciences/Health Foundation, Institute of Psychiatry, Psychology and Neuroscience (loPPN), King's College London, Department of Health Service and Population Research, Centre of Epidemiology and Public Mental Health, London.

\section{Provenance}

Freely submitted; not externally peer reviewed.

DOI: https://doi.org/10.3399/bjgp17X69262

\section{REFERENCES}

1. Brown S. Excess mortality of schizophrenia A meta-analysis. Br J Psych 1997; DOI. https://doi.org/10.1192/bjp.171.6.502.

2. Thornicroft G. Physical health disparities and mental illness: the scandal of premature mortality. Br J Psych 2011; DOI: https://doi. org/10.1192/bjp.bp.111.092718.

3. Chang C-K, Hayes RD, Perera G, et al. Life expectancy at birth for people with serious mental illness and other major disorders from a secondary mental health care case register in London. PLOS ONE 2011; DOI: https://doi.org/10.1371/journal.pone.0019590.

4. Kyrgiou M, Kalliala I, Markozannes G, et al. Adiposity and cancer at major anatomical sites: umbrella review of the literature. BMJ 2017; DOI: https://doi.org/10.1136/bmj.j477.

5. Lawrence D, Hancock K, Kisely S. The gap in life expectancy from preventable physical illness in psychiatric patients in Western Australia: retrospective analysis of population based registers. BMJ 2013; DOI: https://doi. org/10.1136/bmj.f2539.

6. Nielsen RE, Uggerby SA, Jensen SOW, McGrath JJ. Increasing mortality gap for patients diagnosed with schizophrenia over the last three decades - a Danish nationwide study from 1980 to 2010. Schizophrenia Res 2013; DOI: https://doi.org/10.1016/j. schres.2013.02.025.

7. Hayes JF, Marston L, Walters K, et al. Mortality gap for people with bipolar disorder and schizophrenia: UK-based cohort study 2000-2014. Br J Psych 2017; DOI: https://doi. org/10.1192/bjp.bp.117.202606

8. National Institute for Health and Care Excellence. Recommendation 1.3.6.5. In: Psychosis and schizophrenia in adults: prevention and management. CG178. London: NICE, 2014. https://www.nice.org.uk/ guidance/cg178 (accessed 7 Sep 2017)

9. Naylor C, Das P, Ross S, et al. Bringing together physical and mental health: a new frontier for integrated care. London: King's Fund, 2016. https://www.kingsfund.org. uk/sites/files/kf/field/field_publication_file/ Bringing-together-Kings-FundMarch-2016_1.pdf laccessed 1 Sep 2017).

10. Gilbody S, Banham L. Smoking cessation in severe mental illness: what works? Addiction 2010; DOI: https://doi.org/10.1111/j.13600443.2010.02946.x 